\title{
Combinatorics of Genome Rearrangements
}

Guillaume Fertin, Anthony Labarre, Irena Rusu, Éric Tannier and Stéphane Vialette 
(C) 2009 Massachusetts Institute of Technology

All rights reserved. No part of this book may be reproduced in any form by any electronic or mechanical means (including photocopying, recording, or information storage and retrieval) without permission in writing from the publisher.

For information about special quantity discounts, please email special_sales@mitpress.mit.edu

This book was set in Times New Roman and Syntax on 3B2 by Asco Typesetters, Hong Kong. Printed and bound in the United States of America.

Library of Congress Cataloging-in-Publication Data

Combinatorics of genome rearrangements / Guillaume Fertin ... [et al.].

p. $\quad \mathrm{cm} .-$ (Computational molecular biology)

Includes bibliographical references and index.

ISBN 978-0-262-06282-4 (hardcover : alk. paper) 1. Translocation (Genetics)-Mathematical models.

2. Translocation (Genetics) - Data processing. 3. Combinatorial analysis. 4. Genomics-Mathematics.

I. Fertin, Guillaume, 1972- II. Series.

[DNLM: 1. Gene Rearrangement. 2. Genome. 3. Models, Genetic. QU 470 C7312009]

QH462.T7C66 2009

$572.8^{\prime} 77-\mathrm{dc} 22$

2008042152

$\begin{array}{llllllllll}10 & 9 & 8 & 7 & 6 & 5 & 4 & 3 & 2 & 1\end{array}$ 


\section{Contents}

Preface xiii

Acknowledgments $\quad x v$

1 Introduction 1

1.1 A Minimalist Introduction to Molecular Evolution 1

1.2 Birth of the Combinatorics of Genome Rearrangements 4

1.3 Statement of the Problem 6

1.4 Scope of This Survey 7

1.5 Overview of the Models 7

1.6 Organization of the Book 8

\section{DUPLICATION-FREE MODELS: PERMUTATIONS}

2 Genomes as Permutations 13

2.1 The Symmetric Group 13

2.2 The Cycles of a Permutation 14

2.3 Signed Permutations 15

2.4 Distances on Permutation Groups 15

2.4.1 Rearrangements as Generators 16

2.4.2 Invariant Distances 17

2.5 Circular Permutations 18

2.5.1 Classical Circular Permutations 19

2.5.2 Genomic Circular Permutations 19

2.6 First Measures of Similarity between Permutations 20

2.6.1 Breakpoints 20

2.6.2 Common Intervals and Semipartitive Families 21

3 Distances between Unsigned Permutations 25

3.1 Transposition Distance 25

3.1.1 Lower Bounds on the Transposition Distance 26

3.1.2 Upper Bounds 29

3.1.3 Improving Bounds Using Toric Permutations 32 
3.1.4 Easy Cases 33

3.1.5 Approximation Algorithms 34

3.1.6 Conjectures and Open Problems 35

3.2 Prefix Transposition Distance 36

3.2.1 Lower Bounds 37

3.2.2 Upper Bounds 38

3.2.3 Diameter 38

3.2.4 Easy Cases 39

3.2.5 Approximation Algorithms 39

3.2.6 Variant: Insertion of the Leading Element 40

3.3 Reversal Distance 40

3.3.1 Lower Bounds 40

3.3.2 Upper Bounds 43

3.3.3 Easy Cases 43

3.3.4 Computational Complexity 44

3.3.5 Approximation Algorithms 45

3.3.6 Exact Algorithms 46

3.4 Prefix Reversal Distance (Pancake-Flipping) 47

3.4.1 Lower Bounds 47

3.4.2 History 48

3.4.3 Variants 48

3.5 Variants 49

3.5.1 Block Interchange Distance 49

3.5.2 Element Interchange Distances 50

3.5.3 Weighted Reversals 52

3.5.4 Fixed-Length Reversals 54

3.5.5 Bounded Variants 54

3.5.6 Cut-and-Paste 55

3.5.7 Strip Moves 55

3.5.8 Stack-Sorting 56

3.5.9 Tandem Duplications and Random Losses 58

3.5.10 Combined Operations: Reversals and Transpositions

3.6 Relations between Distances on Unsigned Permutations 61

\section{Distances between Signed Permutations 63}

4.1 Conserved Interval Distance 63

4.2 Signed Reversal Distance 64

4.2.1 Reversals 64

4.2.2 The Distance Formula 65

4.2.3 The Scenario of Reversals 67

4.2.4 The Space of All Optimal Solutions 68

4.2.5 Experimental Results 69

4.3 Variants of Sorting by Reversals 69

4.3.1 Perfect Signed Reversal Distance 69

4.3.2 Prefix Reversals (Burned Pancakes) 70

4.3.3 Reversals That Are Symmetric around a Point $\quad 70$ 
4.3.4 Weighted Reversals 71

4.3.5 Fixed-Length Reversals 71

4.4 Combined Operations 72

4.4.1 Reversals and Transpositions 72

4.4.2 Reversals, Transpositions, Transreversals, Revrevs 72

4.5 Double Cut-and-Joins 73

5 Rearrangements of Partial Orders $\mathbf{7 5}$

5.1 Genomes as Partially Ordered Sets 75

5.2 Partially Ordered Sets 75

5.2.1 Basic Definitions 75

5.2.2 Representing Posets 77

5.2.3 Topological Sorting 77

5.3 Constructing a Poset 78

5.4 Reversal Distance 79

5.5 Breakpoint Distance 80

5.5.1 Exact Algorithms 80

5.5.2 Heuristics for Computing the Breakpoint Distance

6 Graph-Theoretic and Linear Algebra Formulations 83

6.1 Simple Permutations and the Interleaving Graph 83

6.2 The Overlap Graph 84

6.3 The Local Complementation of a Graph 85

6.4 The Matrix Tightness Problem 85

6.5 Extension to Sorting by Transpositions 86

6.6 The Intermediate Case of Directed Local Complementation

II MODELS HANDLING DUPLICATIONS: STRINGS

$7 \quad$ Generalities 91

7.1 Biological Motivations 91

7.2 Strings and Rearrangements on Strings 92

7.3 Balanced Strings 94

7.4 How to Deal with Multiple Copies? 95

$8 \quad$ Distances between Arbitrary Strings 97

8.1 The Match-and-Prune Model 98

8.1.1 Breakpoint Distance 100

8.1.2 Signed Reversal Distance 106

8.1.3 Adjacency Similarity 108

8.1.4 Common Intervals Similarity 111

8.1.5 Conserved Intervals Similarity 113

8.1.6 Conserved Intervals Distance 114

8.1.7 MAD and SAD Numbers 118

8.1.8 Heuristics 119 
8.2 The Block Edit Model 123

8.2.1 Block Covering Distance 123

8.2.2 Symmetric Block Edit Distance 126

8.2.3 Large Block Edit Distance 129

8.2.4 String Edit Distance with Transpositions 130

8.2.5 Signed Strings 131

\section{Distances between Balanced Strings 133}

9.1 Minimum Common String Partition Problems 133

9.1.1 Unsigned MCSP 134

9.1.2 Signed MCSP 135

9.1.3 Reversed MCSP 137

9.1.4 Full Breakpoint Distance 138

9.2 Reversal Distance 138

9.2.1 Unsigned Reversals 138

9.2.2 Signed Reversals 141

9.2.3 Sorting by Reversals with Length-Weighted Costs 142

9.2.4 Prefix Reversals on Unsigned Strings (Pancake-Flipping) 144

9.2.5 Reversals of Length at Most $2 \quad 147$

9.3 Unsigned Transpositions 147

9.3.1 Unit Cost Transpositions 147

9.3.2 Length-Weighted Transpositions 150

9.3.3 Restricted Length-Weighted Transpositions 150

9.3.4 Prefix Transpositions 152

9.3.5 Adjacent Swaps 153

9.4 Unsigned Block Interchanges 153

9.4.1 Unit-Cost Block Interchanges 153

9.4.2 Character Swaps 155

9.5 Relations between Distances 157

\section{MULTICHROMOSOMAL MODELS 159}

10 Paths and Cycles 161

10.1 Genomes 161

10.2 Breakpoints 162

10.3 Intervals 163

10.4 Translocation Distance 164

10.4.1 Feasibility 166

10.4.2 Unsigned Genomes 166

10.4.3 Signed Genomes 167

10.4.4 Translocations Preserving Centromeres 168

10.4.5 Variants and Special Cases 169

10.5 Double Cut-and-Joins (2-Break Rearrangement) 170

$10.6 k$-Break Rearrangement 171

10.7 Fusions, Fissions, Translocations, and Reversals 172

10.8 Rearrangements with Partially Ordered Chromosomes 174 
11 Cycles of a Permutation 175

11.1 A Model for Multichromosomal Circular Genomes 175

11.2 A Generalization to Signed Genomes 178

11.2.1 A Different Kind of Signed Permutation 178

11.2.2 The Operations 179

11.2.3 Some Results 179

12 Set Systems and the Syntenic Distance 181

12.1 Introduction 181

12.2 Structural Properties 182

12.2.1 Compact Representation 182

12.3 Lower Bounds 184

12.4 Diameter 185

12.5 Algorithmic Results 185

12.5.1 Syntenic Distance 185

12.5.2 Easy Cases 186

12.6 Conjectures and Open Problems 189

\section{MULTIGENOMIC MODELS 191}

13 Median and Halving Problems 193

13.1 Breakpoint Median 194

13.1.1 Complexity 194

13.1.2 Algorithms 195

13.2 Reversal and DCJ Median 197

13.2.1 Complexity 197

13.2.2 Algorithms 197

13.2.3 Variants 198

13.3 Duplicated Genomes 199

13.3.1 The Double Distance 199

13.3.2 Genome Halving 201

13.3.3 Solving Tetraploidy 202

13.3.4 Guided Halving 202

13.3.5 Genome Halving with Unordered Chromosomes 203

13.4 Other Variants, Generalizations, and Discussion 205

13.4.1 Other Operations 205

13.4.2 More Permutations in the Input 205

13.4.3 Medians and Centers 205

13.4.4 Discussion 206

\section{Rearrangement Phylogenies 207}

14.1 The Large Parsimony Problem 207

14.2 The Large Parsimony Problem with Gene Orders 209

14.2.1 Breakpoint and Reversal Phylogenies on Permutations 209

14.2.2 Variants 211 
14.3 Heuristics for the Breakpoint/Reversal Phylogeny Problem 211

14.3.1 Tree Steinerization 212

14.3.2 Sequential Addition 216

14.3.3 Character Encodings 217

14.4 Variants 220

\section{MISCELLANEOUS 221}

15 Software 223

15.1 Pairwise Rearrangements 223

15.1.1 Unichromosomal Models 223

15.1.2 Multichromosomal Models 225

15.2 Phylogeny Reconstruction and Medians 226

15.2.1 BPAnalysis 226

15.2.2 MGR 226

15.2.3 GRIL 226

15.2.4 GRAPPA 227

15.2.5 MedRbyLS 227

15.2.6 rEvoluzer and amGRP 227

15.2.7 GENESIS 228

\section{Open Problems 229}

16.1 Complexity Issues 229

16.1.1 Hardness 229

16.1.2 Approximability 230

16.1.3 Polynomial Complexity 231

16.2 Diameter 231

16.3 Tightness of Bounds 232

\section{APPENDICES 233}

\section{A Graph Theory 235}

A.1 Undirected Graphs 235

A.1.1 Basic Definitions 235

A.1.2 Paths and Cycles 236

A.1.3 Connectivity 237

A.1.4 Bipartite Graphs 238

A.1.5 Trees and Forests 238

A.1.6 Matching 238

A.1.7 Adjacency Matrix 239

A.2 Directed Graphs 240

A.2.1 Basic Definitions 240

A.2.2 Paths and Cycles 241

A.2.3 Connectivity 241

A.2.4 Directed Acyclic Graphs 241 
B Complexity Theory 243

B.1 The Class NP 243

B.1.1 NP-Optimization Problems: From PTAS to APX 246

B.1.2 NP-Optimization Problems: Beyond APX 250

B.1.3 Parameterized Complexity 250

B.2 Some NP-Complete Problems 252

Glossary 257

Bibliography 263

Index 283 